\title{
British HIV Association national audit on the management of patients co-infected with tuberculosis and HIV
}

\author{
Matthijs Backx, Hilary Curtis, Andrew Freedman and Margaret Johnson on behalf of the British HIV \\ Association (BHIVA) and BHIVA Clinical Audit Sub-Committee
}

\begin{abstract}
This audit aims to compare UK management of tuberculosis (TB)/HIV co-infection with recommended practice and to describe local care arrangements. Services providing HIV care were invited to complete a survey of care arrangements and to review case notes of HIV positive patients aged over 16 who started therapy for active TB between October 2007 and April 2008. Corresponding TB services, if separate, were invited to complete a similar survey. Responses were received from 124 of $170 \mathrm{HIV}$ services, and 18 corresponding TB services. Data were obtained for 236 coinfected patients. Despite some incomplete data, this audit yielded useful findings. Many positive smear results were unacceptably delayed. The TB therapy completion rate fell short of the chief medical officer's (CMO's) $85 \%$ target. Culture confirmation of pulmonary TB met the CMO's $65 \%$ target. A high number of patients were diagnosed with HIV during investigation of TB. Contrary to current guidelines, many services do not routinely test TB patients for HIV.
\end{abstract}

KEY WORDS: audit, clinical networks, HIV, tuberculosis

\section{Introduction}

Although the global incidence of tuberculosis (TB) has started to fall, after reaching its peak in 2004, the total number of cases and deaths continues to rise as the world's population increases. ${ }^{1}$ This is most apparent in Africa where $12 \%$ of the world's population carry $28 \%$ of the global burden of TB. ${ }^{1} \mathrm{HIV}$ infection is the most important risk factor for the development of active TB both through reactivation of latent $\mathrm{TB}$ and the acquisition of exogenous infection. ${ }^{2,3} \mathrm{HIV}$ is thought to be responsible for $8 \%$ of all new adult cases of $\mathrm{TB}$ worldwide though this is likely to be much higher in Africa where $80 \%$ of all TB/HIV co-infections are found. ${ }^{1}$

In 2007, TB/HIV killed 380,000 people worldwide. ${ }^{1}$ Co-infection with HIV/TB negatively impacts on the outcome of both

Matthijs Backx, ${ }^{1}$ specialist registrar in infectious diseases and medical microbiology; Hilary Curtis, ${ }^{2}$ BHIVA audit coordinator; Andrew Freedman, ${ }^{1}$ senior lecturer in infectious diseases and honorary consultant physician; Margaret Johnson, ${ }^{3}$ professor in thoracic medicine and HIV/AIDS

${ }^{1}$ University Hospital of Wales, Cardiff and Vale University Health Board, Cardiff; ${ }^{2}$ British HIV Association, London; ${ }^{3}$ Royal Free Centre for HIV Medicine, Royal Free Hampstead NHS Trust, London diseases. Firstly, HIV leads to increased risk of TB infection after exposure followed by a higher rate of progressive primary and subsequently reactivated disease. Furthermore, extrapulmonary and severe disease, such as meningitis, is more frequent. Finally, TB predisposes to high HIV viral loads and increased rates of progression to AIDS. ${ }^{4,5}$ Combination antiretroviral therapy (cART) has been shown to reduce the risk of developing active TB and TB-related mortality. ${ }^{6-9}$

In the UK in 2008, $6.7 \%$ of adults with TB were co-infected with HIV. ${ }^{10}$ This level is likely to be higher in cities such as London, with large immigrant populations. HIV testing by TB physicians is vital for the identification of co-infected patients and allows interventions such as co-trimoxazole prophylaxis and the introduction of cART. ${ }^{11}$ Despite current efforts, it is estimated that approximately one quarter of the 86,500 people infected with HIV in the UK remain undiagnosed. ${ }^{12}$

In 2005, the British HIV Association (BHIVA) published guidance on the management of TB/HIV co-infection. ${ }^{13}$ Complementary recommendations have since been released by the National Collaborating Centre for Chronic Conditions (NCCCC) entitled Tuberculosis: clinical diagnosis and management of tuberculosis and measures for its prevention and control. ${ }^{14}$ In addition, the Department of Health has released guidance for the control and prevention of $\mathrm{TB} .{ }^{15,16}$

This article investigates how current UK management of TB/HIV co-infection meets with recommended practice by way of a national audit. In addition, the results of a national survey of clinical services aimed at determining how local care is arranged for patients with TB/HIV infection are presented.

\section{Methods}

Services listed on the BHIVA database and thought to provide adult HIV care were contacted by mail and invited to complete an online survey and review case notes of all HIV positive patients aged over 16 who started therapy for active TB during the period October 2007 to April 2008. These HIV services were also asked to contact their corresponding TB services (if separate) and invite them to complete a similar survey. The survey sought to answer two main questions:

- What are the local care arrangements for TB/HIV coinfected patients?

- What are the local policies for HIV testing in patients with $\mathrm{TB}$ ? 
The case-note review was performed between October and December 2008 so that most patients had enough time to complete a full course of anti-TB therapy. Patients receiving chemoprophylaxis for latent TB were excluded, as were patients where TB therapy was commenced but subsequently stopped because of an alternative diagnosis. The following background data were collected: patient age, gender, ethnicity and whether from a high TB prevalence country of origin, the timing of HIV diagnosis, HIV clinic attendance, HIV disease stage at time of TB diagnosis, the site of TB infection and the method of TB diagnosis. Outcomes which were compared to national recommendations are shown in Box 1.

\section{Results}

\section{Surveys of HIV and TB services}

Responses were received from 124 (73\%) of 170 HIV services surveyed, and 18 corresponding TB services. Of the HIV services, 19 (15\%) were fully integrated departments with the same clinical team providing care for mono-infected patients with $\mathrm{TB}$ or with HIV and for co-infected HIV/TB patients. The remaining 105 non-integrated HIV services reported a variety of arrangements for managing HIV/TB co-infection including liaison between HIV and $\mathrm{TB}$ clinicians with each managing their own respective aspects of care ( $68 \mathrm{HIV}$ services, also reported by 16 of the 18 TB services), management of uncomplicated TB cases by HIV clinicians with referral of more complex cases (10) and joint clinics (6).
The majority of services treating TB cases test patients for HIV routinely unless the patient refuses consent $(13(68 \%)$ of 19 integrated services and 10 (56\%) of $18 \mathrm{~TB}$ services). Some others offer testing selectively to patients considered at risk (Table 1). Of the 105 non-integrated HIV services, 17 (16\%) thought that their local TB service only tested selected patients.

\section{Case note review}

Data were obtained on 236 HIV positive patients who started treatment for active TB during October 2007 to April 2008. There were 124 women (52.5\%) and 111 men (47\%). In one case, gender was not stated. The majority of patients were black African $(74.6 \%)$ and a large proportion had originated from high TB prevalence countries $(84.7 \%)$. The most prevalent age group was between 30 and 50 (73.7\%), with fewer patients being aged under 30 years $(13.6 \%)$ or over 50 years $(9.8 \%)$.

Notification of TB was documented for $85(36.0 \%)$ patients and believed to have been done in a further $113(47.9 \%)$. Six $(2.5 \%)$ cases had not been notified, including three with sputum smear positive pulmonary $\mathrm{TB}$, and notification status was unknown for 32 (13.6\%).

Most patients $(185,78.4 \%)$ attended clinic regularly for HIV care, but $21(8.9 \%)$ missed some appointments, six $(2.5 \%)$ had died and $23(9.7 \%)$ had stopped attending including nine $(3.8 \%)$ who were lost to follow-up for HIV (the others having left the UK (9) or transferred care (5)). Information was missing for one.

\section{Box 1. Outcomes compared to national recommendations.}

\begin{tabular}{|c|c|c|}
\hline Outcome & National recommendation & Target \\
\hline TB notification & Public Health Act 1913 & All cases notified by attending physician \\
\hline Culture confirmation of pulmonary TB & Chief medical officer ${ }^{a}$ & $>65 \%$ culture confirmed \\
\hline TB treatment completion & Chief medical officer ${ }^{a}$ & $>85 \%$ treatment completed \\
\hline Sputum smear results & Health Protection Agency ${ }^{b}$ & $\begin{array}{l}\text { Positive results within } 24 \text { hours on six day/week } \\
\text { service; all results in writing within } 72 \text { hours }\end{array}$ \\
\hline
\end{tabular}

Table 1. Policy regarding testing adult tuberculosis (TB) patients for HIV, by numbers (percentage) of different types of site.

$\begin{array}{lll}\begin{array}{l}\text { Non-integrated } \\ \text { HIV services }\end{array} & \begin{array}{l}\text { Integrated HIV/TB } \\ \text { services }\end{array}\end{array}$

Policy

Not applicable*

Test all routinely, unless patient refuses

$13(68) \quad 10(56)$

Offer testing routinely

$2(11)$

4 (22)

Offer testing selectively according to risk

1 (5)

2 (11)

Inconsistent/varies

$1(5)$

0 (0)

Unclear/not answered

2 (11)

$2(11)$

Total

$105(100)$

19 (100)

18 (100)

*17 (16\%) of non-integrated HIV services thought their local TB service only tested selected patients. 
The majority of patients (55.5\%) were diagnosed with HIV prior to their investigation for TB. However, $42 \%$ were diagnosed as part of their investigation for $\mathrm{TB}$ or as a direct result of a TB diagnosis (Table 2). Most patients, 69.1\% $(n=163)$ had advanced HIV disease (CD4 $<200$ cells/ $\mu \mathrm{l}$ ) around the time of starting TB treatment. Some of this group $(n=73)$ were previously diagnosed with HIV, including 41 already on cART, but the majority $(n=90)$ were presenting for the first time with advanced HIV (Fig 1).

Ninety-six patients $(40.7 \%)$ had pulmonary TB. However, extra-pulmonary disease was prevalent, either in combination

\section{Table 2. Characteristics of patients around the time of TB} diagnosis.

\section{Number (\%) of} patients

\section{Timing/circumstances of HIV diagnosis:}

Before seeking care for TB

$131(55.5)$

Of whom, on CART

$70(29.7)$

During investigation leading to TB diagnosis

80 (33.9)

Via routine screening post TB diagnosis

$19(8.1)$

After TB diagnosis but not via routine screening

$4(1.7)$

Not known

$2(0.9)$

CD4 measured nearest to the time of starting

TB treatment, in cells/ $\mu$ l:

0-50

$63(26.7)$

$51-200$

$100(42.4)$

201-350

$36(15.3)$

$>350$

$33(14.0)$

Not known

4 (1.7)

Total

$236(100)$

cART $=$ combination antiretroviral therapy; $\mathrm{TB}=$ tuberculosis.

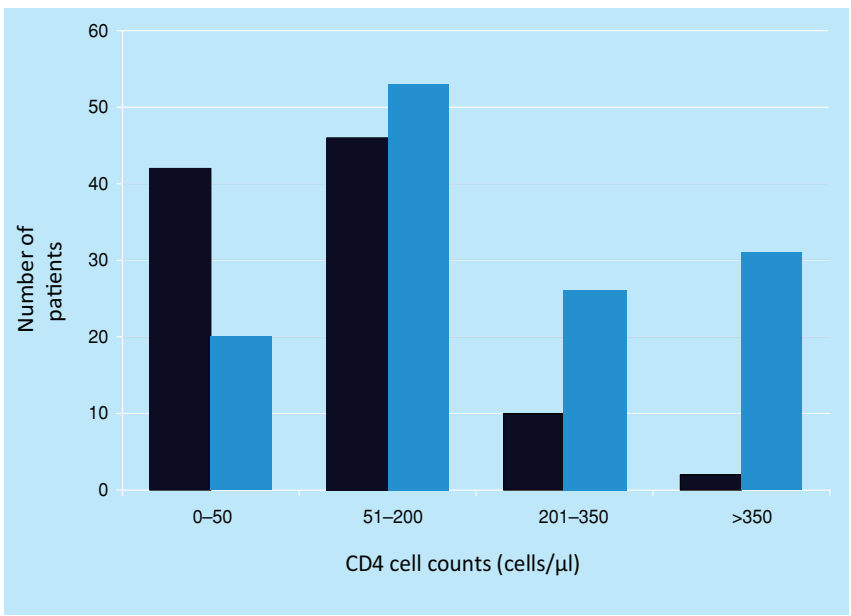

Fig 1. CD4 cell counts in cells $/ \mu \mathrm{l}$ measured nearest to time of tuberculosis (TB) diagnosis. Number of patients diagnosed with HIV at same time as TB (black bars); number of patients previously diagnosed with HIV (blue bars). CD4 cell counts (cells $/ \mu \mathrm{I}$ ). with pulmonary disease (17.4\%) or as an isolated entity (41.9\%).

Most patients $(136,57.6 \%)$ had culture-confirmed TB, including 90 of 137 with pulmonary TB (65.7\%). A further 32 $(13.6 \%)$ were diagnosed via positive microscopy for acid-fast bacilli (AFB) in the absence of culture. In 16 (6.8\%), diagnosis was reached via positive histology, cytology or nucleic acid amplification tests without culture or AFB microscopy. Of the remaining $52(22.0 \%)$ cases, 29 had at least one positive investigation, eg imaging, tuberculin skin testing or raised lymphocytes in cerebrospinal fluid, 20 were purely presumptive diagnoses and information was missing for three.

Among 60 sputum smear positive cases, the result was received on the same or next day as the sample was taken in $27(45 \%)$ and within two to three days for 10 (16.7\%) but took four or more days for 15 (25\%), with data unavailable for eight (13.3\%).

At the time of auditing 144 patients $(61 \%)$ had completed TB treatment without interruption and in $51(21.6 \%)$ treatment was still ongoing. Of $12(5.1 \%)$ patients whose therapy was interrupted, three subsequently completed therapy, and four resumed and were still ongoing. Thus after excluding patients still on therapy, 147 (81.2\%) of 181 patients were known to have completed TB treatment. The remaining patients left the UK while on treatment $(11,4.7 \%)$, transferred their care within the UK $(6,2.5 \%)$, died before completion of therapy $(6,2.5 \%)$ or were lost to follow up $(6,2.5 \%)$. Of nine patients lost to follow up for HIV care, only two completed TB therapy without interruption and in one this was ongoing.

\section{Discussion}

TB remains a major public health concern in the UK with over 9,000 new cases in 2009 and over 450 deaths attributable to TB in 2006. ${ }^{10}$ These figures indicate that TB rates have once again increased, having remained fairly stable between 2005 and 2008.

In 2005, the chief medical officer (CMO) published an action plan for the control of TB in England, ${ }^{13}$ subsequently supported by a toolkit, ${ }^{16}$ with the aim of progressively reducing TB rates within both domestic and immigrant populations. Part of the plan revolved around high quality coordinated services for TB diagnosis, treatment and continuing care, including a recommendation supporting joint services for patients co-infected with TB and HIV. However, we found that the dominant mode of care for such patients was liaison between HIV and TB clinicians with each managing their own aspect of care.

A limitation of the study was the poor response from TB services not providing integrated HIV/TB care, which was likely due to the indirect method of their recruitment. While this limits information about their working practice and interaction with HIV services, it is of concern that a substantial proportion of services did not routinely screen $\mathrm{TB}$ patients for HIV infection.

The action plan also stressed the importance of patient-centred care especially since the treatment of $\mathrm{TB}$ is a long process. This holds particularly true for the HIV/TB co-infected patient 
in whom treatment may be much more complex. Patient retention can be difficult, as a disproportionate number of people with TB have chaotic social circumstances. The case note review found that the majority of co-infected patients attend HIV clinics on a regular basis. However, a small but significant number of patients stopped attending and data suggest a reciprocal relationship between non-attendance for HIV care and non-completion or poor adherence to TB treatment. Similar 'difficult to reach groups' have been identified in previous BHIVA audits. They present a significant challenge as they not only pose a risk to themselves but also to the wider community both from a public health and financial perspective.

The plan calls for continued high quality surveillance to help identify outbreaks, monitor trends, inform policy and monitor the success of the TB programme, which includes the statutory requirement for notification of TB cases. Six out of the 236 audited patients had not been notified and, of these, half had sputum smear positive pulmonary TB. Notification was clearly recorded for only a third of patients, although for some sites this may reflect audit completion using HIV notes without access to TB notes or the use of an electronic notification system. Laboratory reporting of culture-confirmed cases became a requirement in October 2010, while the Health Protection Agency has introduced a web-based enhanced TB surveillance reporting system which aims to improve the completeness, accuracy and accessibility of information. Clearly this needs to be implemented together with increased communication between TB and HIV services.

In association with the British Infection Society and the British Association for Sexual Health and HIV, the British HIV Association published national guidelines on HIV testing in 2008, recognising both the public health impact of undiagnosed HIV infection and the role of HIV co-infection as a risk factor and poor prognostic indicator in TB. These state that HIV testing should be routinely recommended to patients with $\mathrm{TB}$, regardless of perceived risk and on an opt-out basis (where the test is done unless the patient refuses). This study illustrates the importance of HIV screening since a high number of patients were diagnosed with HIV as part of their TB investigations. Disappointingly, many services do not routinely offer HIV testing to all TB patients. This audit did not cover TB patients not known to have HIV but, alarmingly, a separate study found that in 2003-4 over half of TB patients in London were not offered HIV testing. Even among those at highest risk, testing was not universally offered. The overall prevalence of HIV was $9.9 \%$ and only $27 \%$ of those offered testing refused. ${ }^{17}$

Although the majority of patients had advanced disease/AIDS $(C D 4<200)$, more than half of these had not been diagnosed with HIV until they sought care for their TB. This reinforces the fact that a significant proportion of the HIV positive population remains undiagnosed and will potentially present for healthcare with advanced immunosuppression. Clinicians, trusts and commissioners should work together to promote HIV testing in all healthcare settings and reduce the proportion of patients with undiagnosed infection, to benefit both individuals and public health in accordance with guidelines and the CMO's advice. Specifically, commissioners should require universal HIV testing as a key quality indicator for TB services.

It is also of concern that a number of sites in this survey showed unacceptable delays in sputum smear results. The HPA states that laboratories should provide a six-day service during the normal working day for smear examination of appropriate samples, with reporting of positive sputum results within 24 hours. All NHS trusts should require laboratories to comply with this standard. Clinicians should consider conducting further local audits of time to receive these results.

According to the CMO's action plan, TB control is likely to be achieved if patients with suspected TB are seen by a specialist team within two weeks of initial presentation - at least $65 \%$ of pulmonary TB diagnoses are confirmed by laboratory culture of the organism, and treatment outcomes are recorded for all patients with diagnosed TB with at least $85 \%$ completing successfully. Culture is necessary for checking sensitivity to standard treatment regimes, and in this case note review $65.7 \%$ of patients with pulmonary TB were culture confirmed thus achieving this target. However, after excluding patients still on treatment, the TB treatment completion rate was $81.2 \%$. Even if all patients who transferred their care within the UK were assumed to have completed, the target of $85 \%$ would not have been reached. TB treatment centres should continue to monitor and seek to maximise treatment completion rates.

In conclusion, HIV and TB services should provide high quality, coordinated services for the diagnosis, treatment and continuing care of patients. This service must also strive to meet the needs of the individual including those who attend or adhere poorly for TB and/or HIV care.

\section{Acknowledgements}

The BHIVA Audit and Standards Sub-Committee comprises M Backx, C Ball, G Brook, C Carne, D Churchill, H Curtis, A DeRuiter, S Ellis, K Foster, A Freedman, L Garvey, P Gupta, V Harindra, M Johnson, E Monteiro, C O’Mahony, E Ong, K Orton, R Pebody, F Post, C Sabin, A Schwenk, A Sullivan, R Weston, E Wilkins, D Wilson and M Yeomans.

The authors and sub-committee would like to thank clinicians who completed the questionnaire and the Department of Health for financial support for the BHIVA audit programme.

\section{References}

1 World Health Organization. Global tuberculosis control: WHO report 2010. Geneva: WHO, 2010. http://whqlibdoc.who.int/publications/ 2010/9789241564069_eng.pdf www.who.int/tb/publications/global_report/2009/pdf/full_report.pdf

2 Barnes PF, Bloch AB, Davidson PT et al. Tuberculosis in patients with human immunodeficiency virus infection. $N$ Engl J Med 1991;325:1882-4.

3 Selwyn PA, Hartel D, Lewis VA et al. A prospective study of the risk of tuberculosis among intravenous drug users with human immunodeficiency virus infection. N Engl J Med 1989;320:545-50.

4 Toossi Z, Mayanja-Kizza H, Hirsch CS et al. Impact of tuberculosis (TB) on HIV-1 activity in dually infected patients. Clin Exp Immunol 2001;123:233-8. 
5 Badri M, Ehrlich R, Wood R et al. Association between tuberculosis and HIV disease progression in a high tuberculosis prevalence area. Int J Tuberc Lung Dis 2001;5:225-32.

6 United Kingdom Collaborative HIV Cohort Study Group. Tuberculosis among people with HIV infection in the United Kingdom: opportunities for prevention? AIDS 2009;23:2507-15.

7 Lawn SD, Bekker LG, Wood R. How effectively does HAART restore immune responses to Mycobacterium tuberculosis? Implications for tuberculosis control. AIDS 2005;19:1113-24.

8 Girardi E, Palmieri F, Cingolani A et al. Changing clinical presentation and survival HIV-associated tuberculosis after highly active antiretroviral therapy. J Acquir Immune Defic Syndr 2001;26:326-31.

9 Badri M, Wilson D, Wood R. Effect of highly active antiretroviral therapy on incidence of tuberculosis in South Africa: a cohort study. Lancet 2002;359:2059-64.

10 Health Protection Agency. Tuberculosis in the UK. London: HPA, 2005. www.hpa.org.uk/web/HPAwebFile/HPAweb_C/ 1287143594275

11 British HIV Association. UK National Guidelines for HIV Testing 2008. London: HPA, 2008. www.bhiva.org/documents/Guidelines/Testing/ GlinesHIVTest08.pdf

12 Health Protection Agency. HIV in the UK. London: HPA, 2010. www.hpa.org.uk/web/HPAwebFile/HPAweb_C/1287145367237

13 British HIV Association. Guidelines for the treatment of TB/HIV coinfection 2005. London: BHIVA, 2005. www.bhiva.org/documents/ Guidelines/TB/TB_HIV_FINAL2005.pdf
14 National Collaborating Centre for Chronic Conditions. Tuberculosis: clinical diagnosis and management of tuberculosis, and measures for its prevention and control. London: Royal College of Physicians, 2006.

15 Department of Health. Stopping tuberculosis in England: an action plan from the chief medical officer. London: DH, 2004.

www.dh.gov.uk/prod_consum_dh/groups/dh_digitalassets/@dh/@en/ documents/digitalasset/dh_4100860.pdf

16 Department of Health. Tuberculosis prevention and treatment: a toolkit for planning, commissioning and delivering high-quality services in England. London: DH, 2007.www.dh.gov.uk/prod_consum_dh/ groups/dh_digitalassets/@dh/@en/documents/digitalasset/dh_ 075638.pdf

17 Rodger AJ, Story A, Fox Z, Hayward A; London Tuberculosis Nurses Network. HIV prevalence and testing practices among tuberculosis cases in London: a missed opportunity for HIV diagnosis? Thorax 2010;65:63-9.

Address for correspondence: Dr M Backx,

School of Molecular Medical Sciences,

West Block, A Floor, Queen's Medical Centre,

University of Nottingham, Nottingham NG7 2UH.

Email: matthijs.backx@nottingham.ac.uk

\section{Working party reports}

\section{Acute medical care The right person, in the right setting - first time}

Acute medical services and the provision of acute medical care in our hospitals have evolved rapidly over the past decade. Acute medical emergencies are the most common reason for admission to an acute hospital, and acute medicine is the fastest growing medical specialty. Changes to the way acute medical services are delivered has been necessitated by a number of drivers, high among which are patient safety, improved quality of clinical care, clinical governance, and the need to train within the specialty.

Within our hospitals there is a need to reconfigure services to provide more efficient patient access to acute care whenever that need arises. Acutely ill patients require rapid round-the-clock access to senior clinical decision makers, and to a nationally standardised approach to clinical assessment, documentation and illness management.
This report provides practical guidance for the delivery of acute medical services, identifying generic principles that can be configured to meet local needs. It recognises the important role that the multi-professional team plays in delivering a high-quality service. The report updates the 2004 report Acute medicine: making it work for patients and should be read by all those involved in delivering acute medical care and managing acute medical services. -

Published: October 2007 ISBN: 9781860163210

Price: $£ 12.00$ UK, $£ 14.00$ overseas (inc post and packing) 